\title{
Kinematic Analysis of Planar Biomechanical Models using Mixed Coordinates
}

\author{
Ivo Roupa $^{1}$, Sérgio Gonçalves ${ }^{1}, \underline{\text { Miguel Tavares da Silva }}{ }^{1}$ \\ ${ }^{1}$ IDMEC - Instituto de Engenharia Mecânica \\ Instituto Superior Técnico, Universidade de Lisboa \\ Av. Rovisco Pais, No 1, 1049-001, Lisboa, Portugal \\ [ivo.roupa, sergio.goncalves, miguelsilva]@tecnico.ulisboa.pt
}

\begin{abstract}
Inverse Kinematics Analysis (IKA) is a powerful tool to study mechanical and biological systems, since it can be used to adjust the position and orientation of each segment of the model to the experimental data, enabling to achieve reliable and consistent model positions. Optimization-based methods have been successfully applied to perform IKA in intricate biomechanical systems. However, these methods tend to be more complex requiring more computational power and CPU times. This work presents an alternative methodology based on the use of Fully Cartesian Coordinates (FCC) and Mixed Coordinates (MC), alongside with a weighted least square approach to solve the IKA problem. The proposed methodology was applied in the study of a gait movement for a planar full body biomechanical model. The Root Mean Square Error (RMSE) between the experimental and computed positions was calculated both for the proposed method and for a forward kinematic analysis (FKA) carried out with pre-calculated angular drivers, resulting in smaller average differences in the former case (IKA: $0.018 \mathrm{~m}$ FKA: $0.019 \mathrm{~m}$ ). Based on the obtained results it is possible to conclude that the proposed methodology is an accurate, efficient, and reliable approach to perform the IKA of biomechanical models, assuring the kinematic consistency between experimental data and the biomechanical model, and, at the same time, avoiding the usual drawbacks of the use of angular drivers or complex optimization techniques.
\end{abstract}

Keywords: Inverse Kinematics, Fully Cartesian Coordinates, Mixed Coordinates, Least Square Approach.

\section{INTRODUCTION}

Kinematic analysis (KA) is a powerful tool used in the study of biomechanical systems, since it allows for the computation of the orientation of the model segments, trajectory of specific points, angular displacement of joints, among other variables of interest. Two approaches can be used to perform the kinematic analysis of multibody systems, namely, forward kinematics (FK) or inverse kinematics (IK). In the first case, the model is guided using linear and angular drivers calculated in a previous step. Afterwards, the consistent generalized coordinates are obtained by imposing the kinematic constraints that define the model. On the other hand, in IK the position and orientation of each segment is computed by minimizing the difference between the experimental data and a set of points belonging to the model, namely the coordinates of the system or other points of interest. This procedure allows for the fitting of the computational model to the experimental data.

In biomechanical models, FK should be applied with caution due to experimental errors associated to the measurement, in particular soft tissue artifacts (STA) [1]. The STA refers to the motion of the markers on the surface of the body with respect to the underlying bones due to inertial effects, skin deformation and sliding, gravity and muscle contraction [2]. Moreover, STA is task- and subject- dependent, which makes standard filtering techniques ineffective [1][3]. 
Andersen et al. (2009) showed that the use of methodologies to minimize the errors between experimental markers and model points result in significant differences in the kinematic outcomes when compared with standard methods. On its turn, these differences can lead to large errors and inconsistencies during dynamic analysis [3]. Consequently, a method that enables to adjust the model to the output of the system in study is of particular interest for the biomechanics area, since it can minimize the errors associated to the experimental acquisition of anatomical points that constitute the biomechanical model. To address this issue, several methods have been proposed, being the most common based on optimization techniques [4].

In this work, a new approach, based exclusively on kinematic constraints and least-square minimization, is proposed to perform the KA of biomechanical systems. The methodology considers the use of angular coordinates to model the kinematic drivers of the system. These coordinates are referred to as 'mixed coordinates' and complement the set generalized coordinates used by the Fully Cartesian Coordinates (FCC) formulation adopted [5]. This method enables to perform an IK analysis and to determine simultaneously the angular drivers of the model. It allows also for the minimization of the error between experimental and computational points, ensuring a better fit of the model to the experimental data.

In order to assess its applicability and reliability, the MC method was applied in the KA of a gait movement for a planar biomechanical model. The results were posteriorly compared with the ones obtained using FK with angular drivers and with the experimental data.

\section{METHODS}

\subsection{Fully Cartesian Coordinates}

Fully Cartesian Coordinates are a development of Natural Coordinates [4] proposed in [4]-[7] They inherit their two major characteristics, i.e., multibody systems are still described using only the Cartesian coordinates of points and unit vectors, and the kinematic constraint equations are quadratic or linear, thus generating linear or constant contributions to the Jacobian matrix. However, in contrast to the Natural Coordinates formulation, where each rigid body might share points with its adjacent bodies, in FCC each rigid body is defined independently. As so, when applied in planar models, four generalized coordinates are required in its definition, namely the Cartesian coordinates of a point located at its center of mass (CoM) and the Cartesian coordinates of a unit vector representative of its orientation. A detailed description of the FCC formulation can be consulted in [5], [8].

\subsection{Mixed Coordinates}

Within the scope of the experimental analysis of human movement, the traditional KA resorts on the use of experimental trajectories/orientations measured using markers/sensors placed on specific points of the subject in analysis. Since these sensors can move with respect to the anatomical landmarks they refer, non-consistent positions can arise from the KA. MC methodology allows for the minimization of these errors, enabling to determine a position consistent with the defined model and experimental data.

The MC formulation is defined as a combination of FCC with generalized angular coordinates. These coordinates represent the angular degrees-of-freedom (DOFs) of the kinematic pairs of the model, being treated also as generalized coordinates of the system (q). For a general rigid body, the extended generalized coordinates' vector becomes:

$$
\mathbf{q}_{i}=\left\{\begin{array}{lllll}
\mathbf{r}_{i_{x}} & \mathbf{r}_{i_{y}} & \mathbf{u}_{i_{x}} & \mathbf{u}_{i_{y}} & \theta_{i}
\end{array}\right\}^{T}
$$

where $\mathbf{q}_{i}$ is the vector of generalized coordinates of body $i, \mathbf{r}$ is a vector that contains the cartesian coordinates of the center of mass of body $i$, $\mathbf{u}$ is a unit vector that defines body $i$ orientation and $\theta_{i}$ is the angular driver between vector $\mathbf{u}$ from body $i$ and a unit vector belonging to other rigid body (ground body included).

The introduction of MC coordinates leads to an augmented vector of generalized coordinates of the system, since a new angular coordinate is appended, per rigid body, to the already existent vector of generalized coordinates. Therefore, additional kinematic constraints need to be added to the MBS to solve the IK problem. These will be introduced in the form of trajectory 
constraints that map the experimental coordinates of points of interest of the model, such are the markers coordinates, to the rigid body local reference frame, e.g., for a given point $P$ belonging to rigid body $i$, one have:

$$
\mathbf{\Phi}=\mathbf{C}_{P_{i}} \mathbf{q}_{i}-\mathbf{r}_{P}^{*}(t)=\mathbf{0}
$$

where $\mathbf{C}_{\boldsymbol{P}_{\boldsymbol{i}}}$ is a constant transformation matrix that relates the global coordinates of point $P$, obtained experimentally and denoted by $\mathbf{r}_{\boldsymbol{P}}^{*}(\boldsymbol{t})$, with the generalized coordinates of body $i$ [5].

The inclusion of trajectory constraints is of particular relevance on the analysis of biomechanical MBS, since they can relate directly with position of the joints or the trajectory of the markers used in the subject. Moreover, the methodology enables to obtain directly the generalized coordinates of the system, needed to perform the dynamic analysis, and the kinematic angular drivers. This issue is particularly useful, since if the biomechanical model is defined according with the ISB recommendations [9], [10], the methodology provides directly the joints angles, which are one of the variables usually evaluated during the biomechanical analysis.

An important aspect of the MC methodology is that it only requires surgical changes in the structure of the angular driver constraint equations of the FCC formulation to become implemented. More precisely, instead of using angular drivers as inputs to control the DOFs of the system, the angular constraints will now explicitly depend on the newly added angular generalized coordinates. This action will result in an additional contribution to the Jacobian matrix per mix coordinate, i.e. the partial derivative of the angular driver constraint equation with respect to the mix coordinate $\boldsymbol{\theta}$, as well as an additional term in the contribution to the $\boldsymbol{\gamma}$ vector of these constraints (see Table 1 ).

Table 1. Constraint equations (line 1), contributions to Jacobian matrix (line 2) and to the RHS of the velocity and acceleration vectors (lines 3 and 4 ) for two angular constraints (dot and cross product) using MC formulation (note that $\widetilde{\mathbf{u}}$ is a vector orthogonal to $\mathbf{u}$, such that $\mathbf{u} \times \mathbf{v}=\widetilde{\mathbf{u}}^{T} \mathbf{v}$ )

\section{Angular Driver}

\begin{tabular}{ccc}
\hline & Dot Product (DP) & Cross Product (CP) \\
\hline$\Phi(\mathbf{q})$ & $\mathbf{u}^{T} \mathbf{v}-\cos (\theta)$ & $\widetilde{\mathbf{u}}^{T} \mathbf{v}-\sin (\theta)$ \\
$\boldsymbol{\Phi}_{\mathbf{q}}$ & {$\left[\begin{array}{ccccc}\mathbf{v}^{T} & \mathbf{u}^{T} & \sin (\theta)\end{array}\right]$} & {$\left[\begin{array}{lll}-\widetilde{\mathbf{v}}^{T} & \widetilde{\mathbf{u}}^{T} & -\cos (\theta)\end{array}\right]$} \\
$v$ & 0 & 0 \\
$\gamma$ & $-2 \dot{\mathbf{u}}^{T} \dot{\mathbf{v}}-\cos (\theta) \dot{\theta}^{2}$ & $-2 \widetilde{\mathbf{u}}^{T} \dot{\mathbf{v}}-\sin (\theta) \dot{\theta}^{2}$ \\
\hline
\end{tabular}

The addition of the trajectory constraints results in an overconstrained system, as each relation contributes with two equations to the kinematic constraints vector $\boldsymbol{\Phi}$. Different methods can be applied to solve the overconstrained system of equations. The one followed in this work considers the use of a weighted least squares approach (WLS) in tandem with the NewtonRaphson method (NRM). This approach has the main advantage of solving directly the IK problem, without requiring the use of optimization algorithms, such as the one presented in the work of Andersen et al. [11]:

$$
\widehat{\mathbf{q}}_{\mathrm{WLS}}=\left(\boldsymbol{\Phi}_{\mathbf{q}}^{T} \mathbf{Z} \boldsymbol{\Phi}_{\mathbf{q}}\right)^{-1} \boldsymbol{\Phi}_{\mathbf{q}}^{T} \mathbf{Z} \boldsymbol{\Phi}
$$

where $\boldsymbol{\Phi}_{\mathbf{q}}$ is the Jacobian matrix of the system, $\mathbf{Z}$ is the weights matrix and $\boldsymbol{\Phi}$ is the vector of kinematic constraints. 
The use of a weighted approach presents also the advantage of allowing for the definition of different weights for each kinematic constraint. When applied from a biomechanics perspective, the definition of different weights for the trajectory constraints can be used to tune the model to the experimental data, forcing the analysis to track the experimental markers with more relevance or those that are less prone to experimental errors. In a similar way, points characterized by higher levels of STA can be defined with lower weights, being the position of the model to these markers adjusted by the fulfillment of the remaining kinematic constraints which are related with the definition of the topology of the biomechanical model.

\subsection{Implementation of the FCC Formulation with Mixed Coordinates}

The FCC formulation was implemented in an in-house software [5] developed using Python language (v3.7.10) [12]. To solve the IK analysis, the iterative NRM alongside with the WLS routines implemented in the numpy library (numpy.linalg.lstsq) was used. To initially test the method, the nonzero elements of the positive-definite weighting diagonal matrix $\mathbf{W}$ were set to 1.0 as presented in the work of Aguiar et al [13].

The performance of the Mixed Coordinates formulation was evaluated by applying it in the KA of a planar full body biomechanical model. The RMSE between the cartesian coordinates of the experimental points, representing the anatomical joints and other distal points, and the KA outcomes was computed for each joint. In order to explore the computational differences between methods, the same analysis was performed for the results obtained using a FK analysis with angular drivers. Moreover, the time required to perform the analysis and the number of iterations of the NRM were also analyzed. Finally, the model joint angles were statistically compared with the values presented in Winter [14] by calculating the intraclass correlation coefficient (ICC).

\subsection{Experimental Data Acquisition}

The FCC formulation with Mixed Coordinates proposed previously was applied to the study of the gait patterns of one healthy volunteer to evaluate its performance. An informed consent with a detailed explanation of the experimental protocol was signed by the volunteer and the subject's participation on the study was dependent on the acceptance of such informed consent.

Kinematic data was acquired using an optoelectronic motion capture system composed by 14 Infrared ProReflex 1000 cameras (Qualisys $@$, Göteborg, Sweden), with an acquisition frame rate of $100 \mathrm{~Hz}$. Prior to data acquisition, the subject performed an adaptation period to the experimental setup. Afterwards, a 15 seconds static trial was performed to collect the most relevant kinematical model parameters, followed by three gait cycles.

A biomechanical model with 12 segments, based on the one presented in Pamiés et al [15], was implemented. A marker set protocol composed by 68 markers was designed to allow for the driving of all the DOFs of the model. The elbow, wrist, knee, and ankle joint centers were computed as the midpoint of the retro reflective markers placed on lateral and medial bony landmarks of the respective joints. The hip joint center was computed using the regression method proposed by Davis et al. [16]. The trajectories of the elbow and knee markers were reconstructed using clusters of markers placed on the thigh and upper arm.

The coordinates of the markers were filtered using a $2^{\text {nd }}$ order low-pass Butterworth filter with a cut-off frequency of $6 \mathrm{~Hz}$. All necessary calculations were performed using in-house scripts developed in MATLAB (MathWorks@, Natick, MA) and Python 3.7 [12].

\section{RESULTS}

No problems were found, related with the modelling procedure and the convergence of the method, during the IKA of the planar biomechanical model. The average errors associated with the position of the joints were similar in the IK analysis with both formulations. On average, both methodologies present identical accuracy. However, the maximum error is higher when using FKA with angular drivers (FCC). 
A propagation of errors along the gait cycle was observed when using the second method, in particular in the more distal points of the kinematic chains, while the method with MC coordinates presented a more consistent response with the experimental data (see Fig. 1).
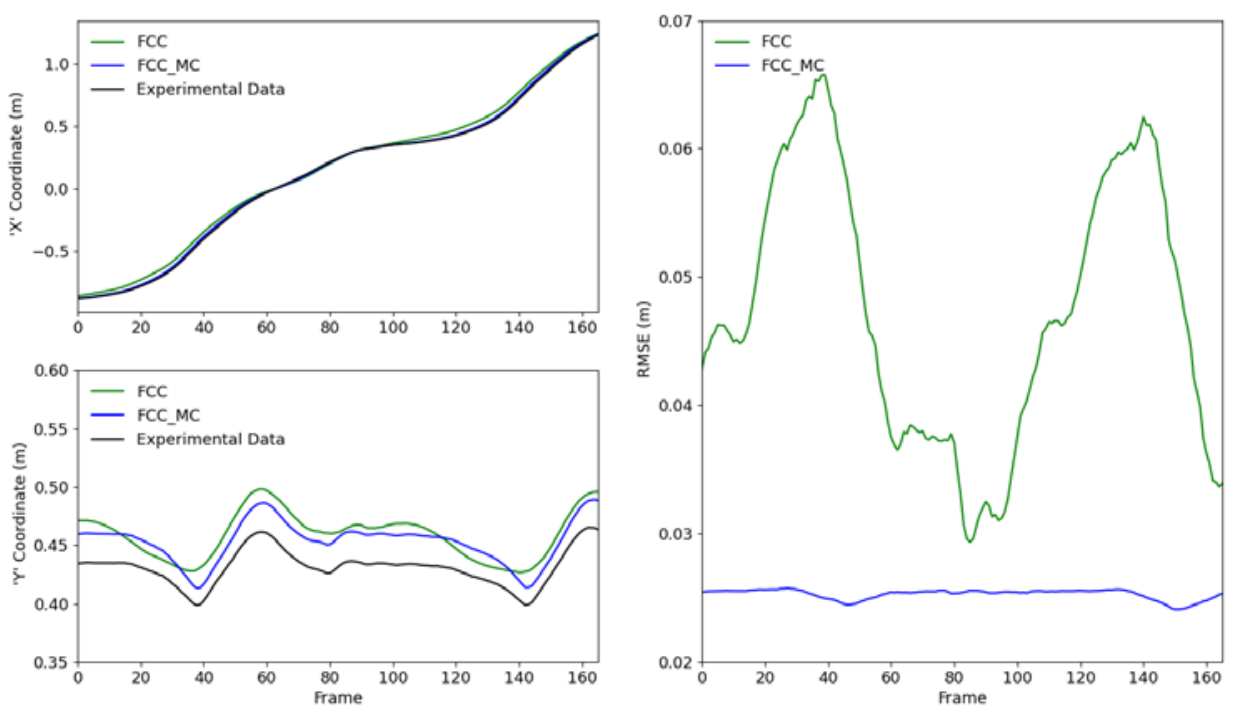

Figure 1. Representation of experimental and computed right knee joint center trajectory throughout the gait trial (upper and lower left figures) and respective Root Mean Square (RMSE) (right figure).

Table 1. Average Root Mean Square Error (RMSE) between the experimental coordinates of each joint center and its estimation based on the consistent generalized coordinates of the model

\begin{tabular}{|c|c|c|c|c|c|c|c|c|c|c|c|c|c|}
\hline \multirow[b]{3}{*}{ Formulation } & \multicolumn{13}{|c|}{ RMSE (m) } \\
\hline & \multirow{2}{*}{$\begin{array}{c}\text { Neck } \\
\text { - }\end{array}$} & \multicolumn{2}{|c|}{ Shoulder } & \multicolumn{2}{|c|}{ Elbow } & \multicolumn{2}{|c|}{ Hip } & \multicolumn{2}{|c|}{ Knee } & \multicolumn{2}{|c|}{ Ankle } & \multirow[t]{2}{*}{ Average } & \multirow[t]{2}{*}{ Max } \\
\hline & & $\mathrm{R}$ & $\mathrm{L}$ & $\mathrm{R}$ & $\mathrm{L}$ & $\mathrm{R}$ & $\mathrm{L}$ & $\mathrm{R}$ & $\mathrm{L}$ & $\mathrm{R}$ & $\mathrm{L}$ & & \\
\hline FCC & 0.0 & 0.009 & 0.037 & 0.011 & 0.008 & 0.012 & 0.011 & 0.032 & 0.028 & 0.026 & 0.028 & 0.018 & 0.037 \\
\hline $\mathrm{FCC}+\mathrm{MC}$ & 0.0 & 0.015 & 0.023 & 0.017 & 0.016 & 0.026 & 0.026 & 0.025 & 0.024 & 0.021 & 0.023 & 0.019 & 0.026 \\
\hline
\end{tabular}

Table 2. Maximum Root Mean Square Error (RMSE) between the experimental coordinates of each joint center and its estimation based on the consistent generalized coordinates of the model

\begin{tabular}{|c|c|c|c|c|c|c|c|c|c|c|c|c|c|}
\hline & \multicolumn{11}{|c|}{ RMSE (m) } & \multirow{3}{*}{ Average } & \multirow{3}{*}{$\operatorname{Max}$} \\
\hline & Neck & \multicolumn{2}{|c|}{ Shoulder } & \multicolumn{2}{|c|}{ Elbow } & \multicolumn{2}{|c|}{ Hip } & \multicolumn{2}{|c|}{ Knee } & \multicolumn{2}{|c|}{ Ankle } & & \\
\hline Formulation & - & $\mathrm{R}$ & $\mathrm{L}$ & $\mathrm{R}$ & $\mathrm{L}$ & $\mathrm{R}$ & $\mathrm{L}$ & $\mathrm{R}$ & $\mathrm{L}$ & $\mathrm{R}$ & $\mathrm{L}$ & & \\
\hline FCC & 0.0 & 0.013 & 0.042 & 0.027 & 0.023 & 0.034 & 0.030 & 0.066 & 0.053 & 0.059 & 0.052 & 0.036 & 0.066 \\
\hline $\mathrm{FCC}+\mathrm{MC}$ & 0.0 & 0.015 & 0.024 & 0.017 & 0.017 & 0.027 & 0.026 & 0.026 & 0.024 & 0.023 & 0.032 & 0.021 & 0.032 \\
\hline
\end{tabular}

Regarding the CPU times, the FKA with FCC took 4.49s and approximately 4 iterations per time frame to obtain the solution using the NRM. On the other hand, the IKA with MC required $12.95 \mathrm{~s}$ and an average of 8 iterations. Although both methods use a least square approach to obtain a solution to solve the problem, these differences could be explained by the higher dimension of the Jacobian matrix in the MC formulation (FK: $\boldsymbol{\Phi}_{\mathbf{q}[58 \times 48]}$, IK: $\boldsymbol{\Phi}_{\mathbf{q}[82 \times 60]}$ ) and the overconstrained nature of that methodology. 


\section{DISCUSSION}

In this work, a methodology based only on kinematic constraints and least square minimization, is presented to compute the IKA of biomechanical systems. When compared to traditional IK techniques, the FCC + MC methodology presents some innovative aspects, such as the simultaneous computation of the generalized coordinates and angular drivers, while assuring the kinematic consistency of the model. This procedure can be achieve without the need of a preprocessing step to obtain a kinematic consistent model from inconsistent experimental data [17].

This feature results from the introduction of trajectory driver equations and angular coordinates in the vectors of kinematic constraints and generalized coordinates of the system. The trajectory driver equations map the experimental coordinates of the points of interest with the associated trajectories and orientations of the rigid segments of the biomechanical model. On its turn, the angular generalized coordinates will represent the angular DOFs of the kinematic pairs of the model, which will be calculated during the IKA. These modifications in the number of generalized coordinates of the system lead to an overdetermined system, which requires a least square approach to find a solution during the IKA. To this purpose an WLS approach was used following the works of Aguiar et al [13], Mantovani et al [18] and Lathrop-Lambach et al. [19]. This method enables to solve directly the linear kinematic constraints, while it simultaneously minimizes the trajectory equations, avoiding the need for complex optimization algorithms.

In the present study, one trajectory constraint was used per joint of the model. However, the method can be easily adapted to consider more points, enabling to fit the model to a cloud of points of interest. The points can also be defined such as the biomechanical model has a direct relation with acquisition protocol used during the experimental trials.

The accuracy of the kinematic reconstruction using the FCC with MC is similar however the RMSE value along the cycle is more stable than when only FCC are used. This is the direct result of the minimization of the distance between the model points and experimental data introduced by the method. These findings are confirmed by the lower RMSE values. A maximum error of $0.066 \mathrm{~m}$ was found for the knee joint, however, on average, all errors are inferior to the error values reported by Ausejo et al [20] as acceptable in motion reconstruction (5 to $25 \mathrm{~mm}$ ).

From a computational point of view, the augmentation of the vector of generalized coordinates due to the inclusion of the angular coordinates and the increase of the number of kinematic constraints results in a complex problem to solve. On average an increase of the number of iterations and time spent per time step was observed in the IKA. However, it is important to note that the processing time spent to obtain the initial angular drivers in the FKA case was not included, while in the proposed IKA approach the reported time already considered all the required steps to perform the kinematic analysis and obtain the joint angles and generalized coordinates of the system. Moreover, despite this increase in the complexity of the problem to solve, the number of iterations per time frame is still reduced, attesting the computational efficiency of the method.

STA is one of the of the most common sources of errors in motion analysis [21], [22]. These inaccuracies can lead to significant errors and inconsistencies during the computation of the kinematic outcomes and, consequently, also on the dynamic results [23]-[25]. To overcome this problem, several methods have been proposed to reduce these experimental errors, being the most commons based on optimization techniques [26]. A different methodology is followed in this work, as only kinematic constraints and trajectory controls are applied. This approach enables to adjust the coordinates of the joints and other distal points simultaneously based on the minimization of distances between these and the equivalent experimental points and the kinematic constraints.

Besides reducing possible errors introduced by the STA, this adjustment of the experimental data to the biomechanical model enables to correct also errors introduced by the placement of markers on positions different from the ones defined in the begin of the analysis (e.g., fall of markers during the trial). Since the biomechanical model is defined using kinematic constraints based on measures acquired during the statical trial, errors in the placement of the markers will be partially corrected by the fulfilling of the kinematic constraints that define the rigid bodies and joints. 
In addition, IKA avoids one of the main drawbacks of the typical FKA based on angular drivers, namely the propagation of errors along the kinematic chain, i.e., experimental errors will be passed continuously to the child bodies, resulting, in general, in higher distances between the experimental and model points in the distal joints.

From a modelling perspective, the use of the FCC with MC presents also other advantages, as the points used in the trajectory constraints can be defined such as they have direct relation with markers used on the common marker set protocols. Moreover, due to the simplicity of the FCC in the modelling of biomechanical models, the angular drivers can be defined to match the normal convention of the joint angles, computing them directly while solving the IKA. These characteristics are of particular relevance for the biomechanics community. By providing a methodology that computes simultaneously the joint angles and has a direct relation with the acquisition protocols, the pre-processing of the experimental data is simplified, facilitating its use.

Despite its promising results, the present study was based on a minimal dataset. Future studies with larger data samples should be carried out, as well as an extension to 3D models, to further validate the methodology.

\section{CONCLUSIONS}

This study presents a methodology to perform inverse kinematic analysis of multibody systems based on FCC with MC. This approach, based solely on kinematic constraints and a WLS method, allows for the determination of the consistent generalized coordinates, while simultaneously minimizing the error between the experimental and computed data.

In the presented case, the accuracy of the kinematic reconstruction using the FCC with MC is significantly higher than when only FCC are used, and therefore, it is possible to conclude that the proposed methodology provides an accurate, efficient, and reliable approach to perform kinematic analysis of human motion, avoiding the problems related with the use of kinematic drivers. Moreover, the IKA considering the FCC+MC formulation presents the advantage of computing the angular drivers that rule the system, without a pre-processing step.

\section{REFERENCES}

[1] J. Fuller, L.-J. Liu, M. C. Murphy, and R. W. Mann, “A comparison of lower-extremity skeletal kinematics measured using skin- and pin-mounted markers,” Hum. Mov. Sci., vol. 16, no. 2-3, pp. 219-242, Apr. 1997, doi: 10.1016/S0167-9457(96)00053-X.

[2] J. P. Holden, J. A. Orsini, K. L. Siegel, T. M. Kepple, L. H. Gerberc, and S. J. Stanhope, "Surface movement errors in shank kinematics and knee kinetics during gait ',” vol. 5, pp. 217-227, 1997.

[3] M. S. Andersen, M. Damsgaard, B. MacWilliams, and J. Rasmussen, “A computationally efficient optimisation-based method for parameter identification of kinematically determinate and over-determinate biomechanical systems," Comput. Methods Biomech. Biomed. Engin., vol. 13, no. 2, pp. 171-83, Jan. 2010, doi: 10.1080/10255840903067080.

[4] G. Jalon and E. Bayo, Kinematic and Dynamic Simulation of Multibody Systems: The Real-Time Challenge. New York: Springer Verlag, 1993.

[5] I. Roupa, S. B. Gonçalves, and M. Tavares da Silva, "Dynamic Analysis of Planar Multibody Systems with Fully Cartesian Coordinates," Proceedings of International Conference on Multibody System Dynamics. Lisbon, Portugal, June 24-28, 2018.

[6] M. T. Gameiro, P., Silva, "Modelação e Simulação Sistemática em Coordenadas Cartesianas Totais de Sistemas Multicorpo,” Actas do Congresso de Métodos Numéricos em Engenharia. Porto, Portugal, Junho 13-15, 2007.

[7] M. Silva and J. Ambrósio, "Nova Abordagem á Modelação de Sistemas de Corpos Múltiplos Utilizando Coordenadas Naturais," Actas do VII Congresso de Mecânica Aplicada e Computacional. Évora, Portugal, Abril 14-16, 2003.

[8] P. Gameiro and M. Tavares da Silva, "A systematic approach to the simulation of multibody systems with natural coordinates," Actas da ECCOMAS Thematic Conference on Multibody Dynamics 2005. Madrid, Spain, June 21-2. 2005. 
[9] G. Wu et al., "ISB recommendation on definitions of joint coordinate system of various joints for the reporting of human joint motion — Part I: Ankle, Hip, and Spine,” J. Biomech., vol. 35, no. 4, pp. 543-548, Jun. 2002, doi: 10.1006/rtph.2002.1549.

[10] G. Wu et al., "ISB recommendation on definitions of joint coordinate systems of various joints for the reporting of human joint motion - Part II: Shoulder, Elbow, Wrist and Hand,” J. Biomech., vol. 38, no. 5, pp. 981-992, 2005, doi: 10.1016/j.jbiomech.2004.05.042.

[11] M. S. Andersen, M. Damsgaard, and J. Rasmussen, "Kinematic analysis of over-determinate biomechanical systems,” Comput. Methods Biomech. Biomed. Engin., vol. 12, no. 4, pp. 371-384, 2009, doi: 10.1080/10255840802459412.

[12] G. Van Rossum and F. L. Drake, “Python \& C API Reference Manual,” no. March, p. 151, 2006.

[13] L. Aguiar, C. Andrade, M. Branco, R. Santos-Rocha, F. Vieira, and A. Veloso, "Global optimization method applied to the kinematics of gait in pregnant women,” J. Mech. Med. Biol., vol. 16, no. 6, 2016, doi: 10.1142/S0219519416500846.

[14] D. Winter, The Biomechanics and Motor Control of Human Gait: Normal, Elderly and Pathological, 2nd ed. Waterloo, Ontario, Canada: University of Waterloo, 1991.

[15] R. Pàmies-Vilà, J. M. Font-Llagunes, J. Cuadrado, and F. J. Alonso, “Analysis of different uncertainties in the inverse dynamic analysis of human gait,” Mech. Mach. Theory, vol. 58, no. January 2019, pp. 153-164, 2012, doi: 10.1016/j.mechmachtheory.2012.07.010.

[16] R. B. Davis, S. Ounpuu, D. Tyburski, and J. R. Gage, “A gait analysis data collection and reduction technique,” Hum. Mov. Sci., vol. 10, no. 5, pp. 575-587, 1991, doi: 10.1016/01679457(91)90046-Z.

[17] M. Tavares da Silva, "Human Motion Analysis using Multibody Dynamics and Optimization Tools,” Universidade Técnica de Lisboa - Instituto Superior Técnico, 2003.

[18] G. Mantovani and M. Lamontagne, "How Different Marker Sets Affect Joint Angles in Inverse Kinematics Framework,” J. Biomech. Eng., vol. 139, no. 4, 2017, doi: 10.1115/1.4034708.

[19] R. L. Lathrop-Lambach et al., "Evidence for joint moment asymmetry in healthy populations during gait,” Gait Posture, vol. 40, no. 4, pp. 526-531, 2013, doi: 10.1016/j.gaitpost.2014.06.010.

[20] S. Ausejo, Á. Suescun, and J. Celigüeta, “An optimization method for overdetermined kinematic problems formulated with natural coordinates,” Multibody Syst. Dyn., vol. 26, no. 4, pp. 397-410, 2011, doi: 10.1007/s11044-011-9263-X.

[21] A. Cappello, R. Stagni, S. Fantozzi, and A. Leardini, "Soft Tissue Artifact Compensation in Knee Kinematics by Double Anatomical Landmark Calibration: Performance of a Novel Method During Selected Motor Tasks,” vol. 52, no. 6, pp. 992-998, 2005.

[22] R. Stagni, S. Fantozzi, A. Cappello, and A. Leardini, "Quantification of soft tissue artefact in motion analysis by combining 3D fluoroscopy and stereophotogrammetry: a study on two subjects.," Clin. Biomech. (Bristol, Avon), vol. 20, no. 3, pp. 320-9, Mar. 2005, doi: 10.1016/j.clinbiomech.2004.11.012.

[23] V. Camomilla, A. Cereatti, A. G. Cutti, S. Fantozzi, R. Stagni, and G. Vannozzi, "Methodological factors affecting joint moments estimation in clinical gait analysis: A systematic review," Biomed. Eng. Online, vol. 16, no. 1, pp. 1-27, 2017, doi: 10.1186/s12938-017-0396-x.

[24] M. P. T. Silva and J. A. C. Ambrósio, "Kinematic data consistency in the inverse dynamic analysis of biomechanical systems,” Multibody Syst. Dyn., vol. 8, no. 2, pp. 219-239, 2002, doi: 10.1023/A:1019545530737.

[25] M. P. T. Silva and J. A. C. Ambrósio, "Sensitivity of the results produced by the inverse dynamic analysis of a human stride to perturbed input data,” Gait Posture, vol. 19, no. 1, pp. 35-49, 2004, doi: 10.1016/S0966-6362(03)00013-4.

[26] M. Begon, M. S. Andersen, and R. Dumas, "Multibody Kinematics Optimization for the Estimation of Upper and Lower Limb Human Joint Kinematics: A Systematized Methodological Review,” J. Biomech. Eng., vol. 140, no. 3, 2018, doi: 10.1115/1.4038741. 